\title{
Synchronous imaging of coherent plasma fluctuations
}

\author{
S. R. Haskey, ${ }^{\text {a) }}$ N. Thapar, B. D. Blackwell, and J. Howard \\ Plasma Research Laboratory, Research School of Physics and Engineering, \\ The Australian National University, Canberra, ACT 0200, Australia
}

(Received 13 December 2013; accepted 1 March 2014; published online 20 March 2014)

\begin{abstract}
A new method for imaging high frequency plasma fluctuations is described. A phase locked loop and field programmable gate array are used to generate gating triggers for an intensified CCD camera. A reference signal from another diagnostic such as a magnetic probe ensures that the triggers are synchronous with the fluctuation being imaged. The synchronous imaging technique allows effective frame rates exceeding millions per second, good signal to noise through the accumulation of multiple exposures per frame, and produces high resolution images without generating excessive quantities of data. The technique can be used to image modes in the MHz range opening up the possibility of spectrally filtered high resolution imaging of MHD instabilities that produce sufficient light fluctuations. Some examples of projection images of plasma fluctuations on the H-1NF heliac obtained using this approach are presented here. ( 2014 AIP Publishing LLC. [http://dx.doi.org/10.1063/1.4868504]
\end{abstract}

\section{INTRODUCTION}

MHD instabilities such as Alfvén eigenmodes, ${ }^{1}$ tearing modes, and sawteeth can limit achievable plasma parameters, and cause detrimental disruptions. ${ }^{2,3}$ The identification and control of these modes relies on accurate measurements of their internal structure, and comparison with modelling. Soft $\mathrm{x}$-ray arrays, and ECE, are often used to provide line of sight measurements which can be tomographically inverted. However, these arrays offer a limited number of line-integrated samples, so a priori assumptions are required for useful inversion. Optical CCD cameras have been deployed for imaging and tomography of plasma structures, but frame rates are often limited by plasma light fluxes to around 100 frames per second. ${ }^{4,5}$

High resolution imaging with fast framing cameras has been used to image the evolution of filament structures during edge localised modes ${ }^{6}$ as well as filaments/blobs near the last closed flux surface. ${ }^{7}$ Additionally, fast 2D imaging of bremsstrahlung emissions from modes with frequencies up to $\approx 10 \mathrm{kHz}$ has been achieved using a fast framing camera. ${ }^{8-11}$ These approaches require sufficient light emission from the plasma per exposure, generate large amounts of data and are band-limited to resolving fluctuations with period of order twice the exposure time.

The synchronous imaging (SI) technique is quite general and provided enough signal is available, can be used to image mode related fluctuations in bremsstrahlung, ${ }^{8}$ soft x-rays (using a scintillator plate to convert the soft $\mathrm{x}$-rays to visible light ${ }^{12}$ ), or spectral lines using interference filters. The use of an intensified camera with gated exposure phase-locked to the mode fluctuation period allows imaging of quasi-coherent fluctuations with frequencies from the low $\mathrm{kHz}$ to $\mathrm{MHz}$ range. This opens up the possibility of directly imaging MHD instabilities that produce sufficient light fluctuations which can have frequencies in the hundreds of $\mathrm{kHz}$, without requiring bright emission from the plasma. The key to the technique is

\footnotetext{
a) shaun.haskey@anu.edu.au
}

the generation of camera timing pulses that are phase-locked to the mode. This allows the camera to accumulate enough charge to produce a high fidelity image by accumulating many phase-locked gated exposures. By changing the phase of the clock pulses between frames, the process can be repeated and a phase-resolved set of projection images spanning a full cycle of the mode is generated. With modern CCD's, these images represent millions of lines of sight and can provide sufficient information to obtain a reliable tomographic reconstruction of the fluctuation structure.

The motivation for this work is to better understand the nature of global MHD wave modes observed in the $\mathrm{H}$ 1NF heliac. ${ }^{13}$ These fluctuations exhibit a systematic relationship between mode structure and frequency and the magnetic configuration. ${ }^{14-17}$ The ultimate goal of the work is to image the mode-associated perturbations in the emission of various helium atomic transition lines and to use this information, in conjunction with collisional radiative models, ${ }^{18,19}$ to infer the structure of the electron density and temperature perturbations for comparison with theoretical predictions. ${ }^{20,21}$

This paper is organised as follows. Section II describes the synchronous imaging system, including details of the hardware, system performance, and timing considerations. Section III shows first results of the synchronous imaging on $\mathrm{H}-1 \mathrm{NF}$ using emission from singly charged carbon impurity ions at $514 \mathrm{~nm}$.

\section{THE SYNCHRONOUS IMAGING TECHNIQUE}

\section{A. Description of hardware}

An overview of the SI approach is shown in Figure 1. A low latency signal from another diagnostic provides the reference signal (Figure 1(a)). For magnetohydrodynamic waves, an obvious choice is the signal from one of an array of magnetic pickup coils which are often used to detect and analyse plasma perturbations. ${ }^{22}$ This signal is amplified and appropriately filtered before providing the reference input to the phase comparator section of an analog PLL (Figure 1(b)). We have 


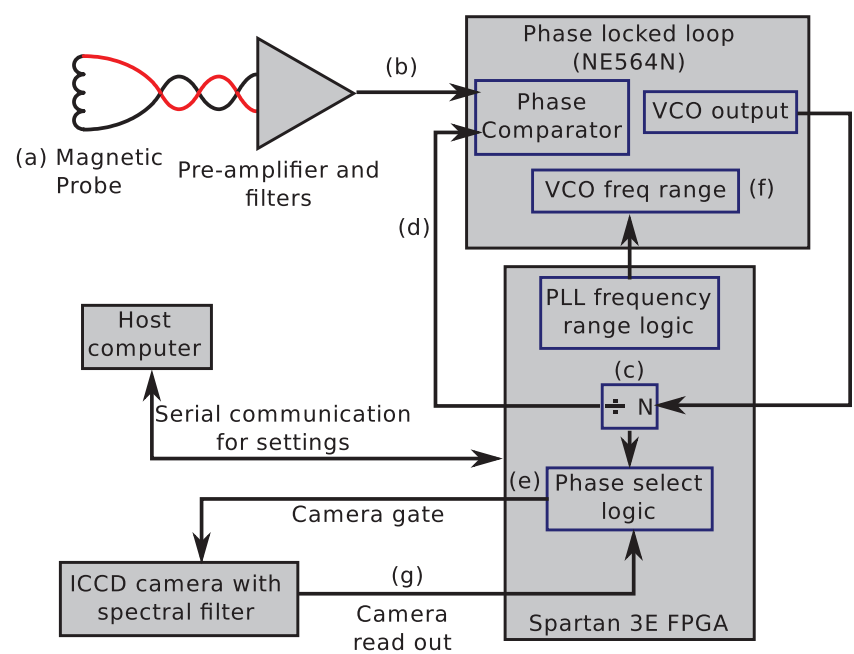

FIG. 1. Overview of the SI system consisting of a magnetic probe which provides the reference signal, a phase locked loop (PLL) for locking to the mode frequency, and field programmable gate array (FPGA) for the logic and generating the intensified CCD (ICCD) gate trigger signals.

used the NE564N PLL which has guaranteed operation up to $50 \mathrm{MHz}$.

A Xilinx Spartan 3E field programmable gate array (FPGA) generates the ICCD gate triggers and provides the logic to select the relative phase that is being imaged for each camera frame. It does this by acting as a clock divisor for the voltage controlled oscillator (VCO) output of the PLL. The divisor, $N$, is set to the number of phases to be captured across the fluctuation cycle (Figure 1(c)). For example, if we want 16 frames (an image for every $\pi / 8$ radians), then $N=16$. The VCO clock divided signal is fed back into the phase comparator input of the PLL (Figure 1(d)). This forces the VCO clock output to be upshifted to $N$ times the frequency of the input reference signal, and it will track the changes in the reference signal as the frequency of the mode varies. Using this high frequency clock, the FPGA can output a ICCD gate trigger every $N$ VCO clock cycles. These triggers will occur at the same phase with respect to the reference signal (Figure 1(e)). Changing the initial count changes the phase when the gate trigger occurs with respect to the reference signal allowing the ICCD to be repetitively gated at the phase of choice.

The frequency range that the PLL can lock, and remain locked is determined by a capacitance which the FPGA controls by switching an array of capacitors in and out (Figure 1(f)). This is generally set before a plasma pulse, because the frequency of the mode can be generally anticipated, although the possibility of actively changing the frequency range during a shot exists. The camera read output is provided as an input to the FPGA (Figure 1(g)), so that the phase that is being imaged can be changed after the camera is finished downloading the previous image to a host computer (or local memory). This allows several images at different phases to be acquired in quick succession, limited by the image transfer rate and the plasma pulse duration. A host computer communicates with the FPGA over a serial link ahead of a shot, and uploads the number and sequence of phases to be imaged along with the desired frequency range of the VCO.

\section{B. Performance of the phase locked loop}

The performance of the PLL and FPGA is demonstrated in Figure 2. A magnetic probe from the helical Mirnov array on $\mathrm{H}-1 \mathrm{NF}^{22}$ is used as the reference signal for the PLL. The top plot shows a coherent mode oscillation in the probe signal, while the PLL and FPGA generated ICCD gate triggers derived from this reference signal are over-plotted. The bottom plot shows the phase obtained from the Hilbert transform of the probe signal. The times of the camera gates as marked by the solid dots clearly demonstrate a high mutual coherence.

Typical data for an experimental pulse are shown in Figure 3. Here the FPGA was programmed to step through four different phases during a plasma pulse on H-1NF. The top plot shows the spectrogram of the reference magnetic probe signal. There is a strong coherent mode from $55 \mathrm{~ms}$ onwards at a frequency close to $60 \mathrm{kHz}$. The center plot shows the ICCD gate trigger signals and ICCD readout signals. Commencing at $50 \mathrm{~ms}$, the first frame accumulates 30 exposures. Once the exposures are finished, the camera waits a fixed amount of time to allow the phosphor to decay, and then starts to download the frame to a host computer (signified by the high camera readout signal). The phosphor decay time is due to the intensifier, and its value can vary depending on the phosphor used (for example, P43, P46, and P47 phosphors have decay times of $3 \mathrm{~ms}, 2 \mu \mathrm{s}$ and $0.5 \mu \mathrm{s}$, respectively). As soon as the camera has finished reading out, the FPGA switches to the next programmed phase, and produces the desired camera gate pulse sequence. The process repeats itself for as many different phases and images as required. The bottom plot shows the phase of the probe signal at the camera gate time. The phases of the 2nd, 3rd, and 4th images are clustered at the pre-programmed phases as required. The phases for the first image appear to be random because the mode amplitude is not sufficient to lock the PLL at $50 \mathrm{~ms}$.

Analysis of phase jitter of the ICCD gate triggers for 16 frames obtained during an experimental sequence is
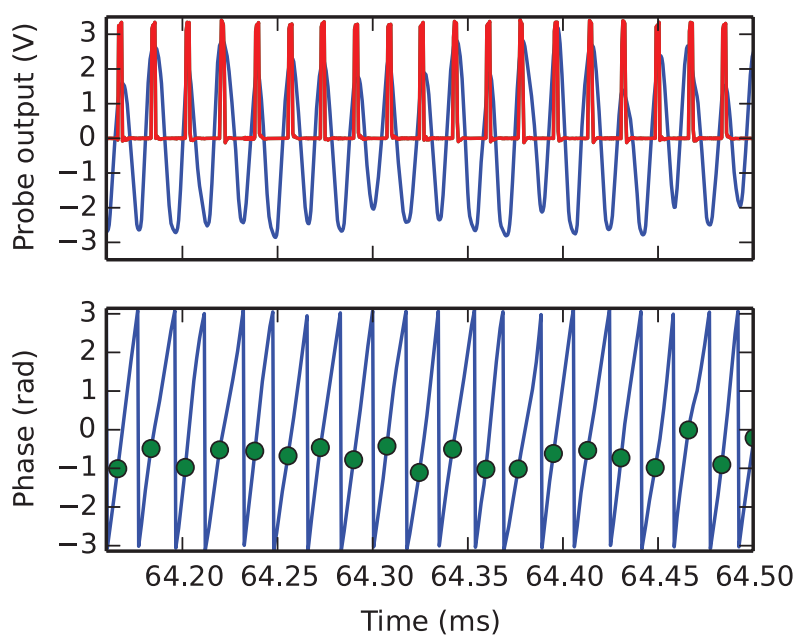

FIG. 2. Performance of the phase locked loop during a single shot. Top: Magnetic probe output and the camera gating trigger signal generated by the FPGA (red pulses). Bottom: Phase of the mode as a function of time calculated using the argument of the Hilbert transform of the probe signal. The dots represent camera gating times, which are all at approximately the same phase showing a good lock to the magnetic probe signal. 

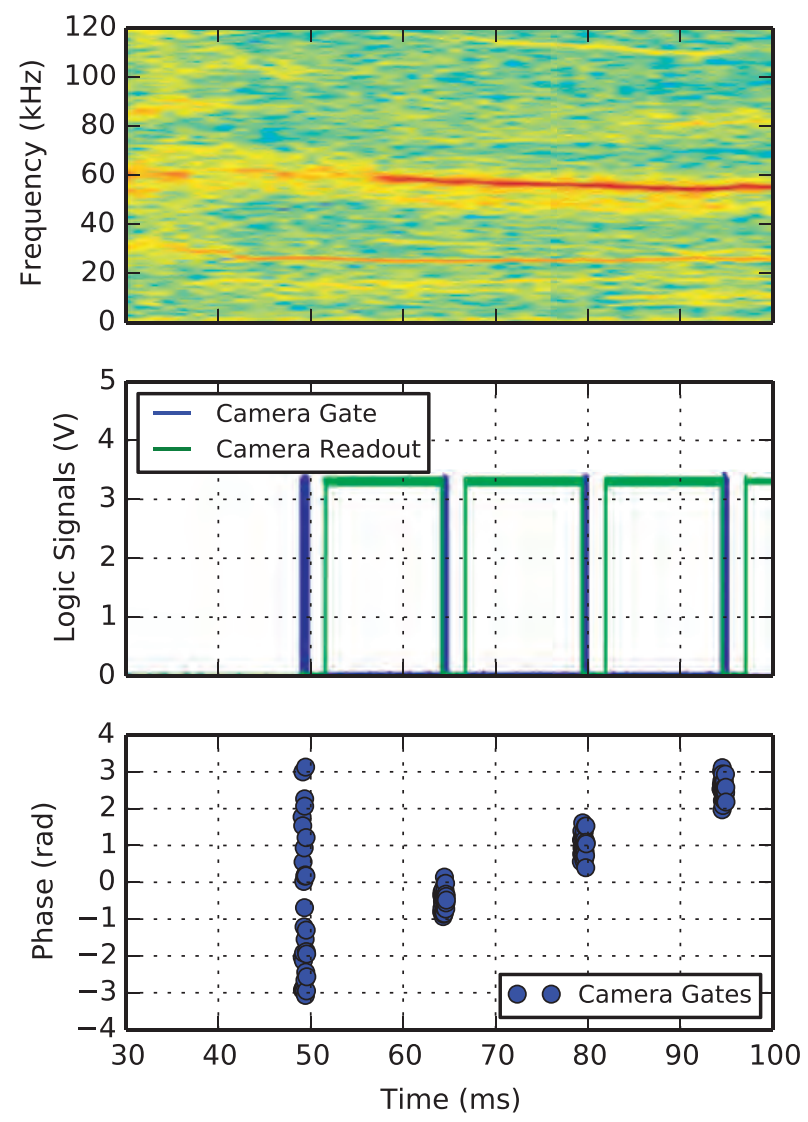

FIG. 3. Top: Spectrogram of the reference magnetic probe signal. Middle: ICCD gate trigger signals (too closely spaced to separate on this scale), and ICCD readout signal. Bottom: Phase of the mode at the ICCD gate trigger times showing consistent phase. The first frame at $50 \mathrm{~ms}$ has random phasing because there is no mode to lock to until $55 \mathrm{~ms}$.

presented in Figure 4. The observed mean and standard deviation of the mode phase at the time of the ICCD gating trigger for each frame demonstrates that the system can successfully track the mode phase with small jitter. Simple analysis, by assuming that phase jitter causes an averaging over a portion of a sine wave shows that the phase jitter has a

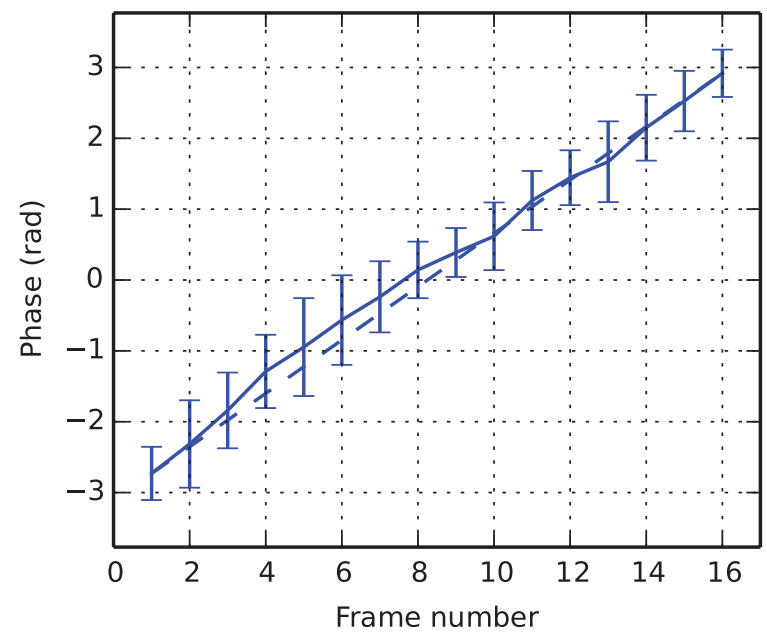

FIG. 4. Performance of the phase locked loop for a 16 frame experimental sequence. The mean phase for each frame is shown along with the standard deviation. The phase shifts between frames is $\pi / 8$ as expected for 16 frames. The dashed line is the ideal case with perfect spacing. minimal effect. For example, phase jitter of the order 1 radian peak to peak, like that shown in Figure 4, causes a smoothing that results in a small 5\% reduction in the amplitude of the signals. Because the phase is a periodic quantity, the maximum likelihood estimates of the mean and standard deviation are calculated as $\hat{\mu}=\arg (R)$, and, $\hat{\sigma}=\sqrt{-2 \ln (|R|)}$, where $R=\frac{1}{n} \sum_{j=1}^{n} \exp \left(i x_{j}\right), x_{j}$ represent the phases, $i=\sqrt{-1}$ and $n$ is the number of gate triggers for the particular phase. ${ }^{23,24}$

\section{Timing considerations}

Using this technique it is possible to obtain extremely high effective frame rates. For example, a conventional fast framing camera would need to operate at 16 million fps to image a $1 \mathrm{MHz}$ mode with 16 frames per cycle, generating enormous quantities of data in the process. A camera operating at this speed would require an exceptionally strong light source especially if interference filters are being used to isolate spectral lines. Without strong gain, a plasma fluctuation is highly unlikely to be able to produce sufficient light. The ability to accumulate multiple exposures as in the SI technique allows one to obtain high fidelity images, even in relatively light poor situations.

The main limitation of the technique is the requirement that the coherent oscillation and plasma conditions remain stable over the duration of the imaging sequence, or alternatively, that the mode and plasma conditions are reproducible enough that the phase-resolved sequence can be acquired over multiple discharges. The time required to acquire the data, $T$, is approximately:

$$
T=N^{2} t_{e}+N\left(t_{r}+t_{d}\right),
$$

where $N$ is the number of phases we wish to acquire, $t_{e}$ is the exposure time required to obtain enough counts, $t_{r}$ is the readout time for each frame, and $t_{d}$ is the decay time for the phosphor. It is worth noting that the time required to acquire the data is independent of the mode frequency. Here we choose the gate time to be approximately equal to the wave period divided by the number of frames to be acquired to obtain the phase resolved sequence ( $3.5 \mu$ s was used for the data shown in Sec. III).

As the gate time increases compared with the mode period, the SI signal level of the mode is reduced. An approximation to the loss of contrast (found by integrating a sine wave over a fixed interval) is given by $1-\operatorname{sinc}(\tau)$ where $\tau$ is the gate time as a proportion of the wave period. Using this approximation, gate times of $1 / 16,1 / 4$, and $1 / 2$ of the wave period result in $1 \%, 10 \%$, and $36 \%$ reduction in the mode signal strength, respectively. The minimum available gating time on an ICCD camera such as the Princeton Instruments PiMAX4 is of the order 3 ns, so this does not limit the technique.

Calculating the required exposure time $\left(t_{e}\right)$ depends on many variables related to the camera setup, intensifier gain and plasma parameters. We set the exposure time, and number of sequence phases empirically, so that there are sufficient photo-counts to resolve the fluctuation above shot noise while ensuring that the camera does not saturate and that the mode activity remains steady throughout the exposure sequence. 
For applications on $\mathrm{H}-1 \mathrm{NF}$ imaging $514 \mathrm{~nm}$ CII light, $t_{e}$ was of the order $350 \mu$ s when using a relatively low intensifier gain and $4 \times 4$ pixel binning on a $1024 \times 1024$ CCD array. This means the first term in Eq. (1) is $90 \mathrm{~ms}$ for 16 frames. In many situations this time is sufficiently short for the mode and plasma conditions to remain stable. The second term in Eq. (1) depends on the camera capabilities. The PiMAX 4 camera with $\mathrm{P} 43$ phosphor used for the data in this paper has a readout time of $13 \mathrm{~ms}$ for $256 \times 256$ resolution and a phosphor decay time of $3 \mathrm{~ms}$. This gives $256 \mathrm{~ms}$ for the second term in Eq. (1) which is significantly larger than the first term. This is evident in Figure 3 where the ICCD readout time is substantially greater than the total gate time. On $\mathrm{H}-1 \mathrm{NF}$, the discharges are reproducible, $\approx 100 \mathrm{~ms}$ in length and the time between shots is roughly a minute so separate discharges were used to acquire each of 16 independent phase-locked frames. On longer pulse plasma devices, and provided the light fluxes are sufficiently high, and the structure sufficiently steady, it is feasible to obtain an entire phase-resolved sequence within a single discharge.

\section{IMAGING RESULTS FROM H-1NF}

The projection images shown here were obtained using a Princeton Instruments PiMAX 4 intensified gated camera. The experimental geometry is illustrated in Figure 5. The camera views the plasma radially in a gap between the toroidal field coils. The lines of sight therefore cover the poloidal cross-section of the H-1NF device and extend over a

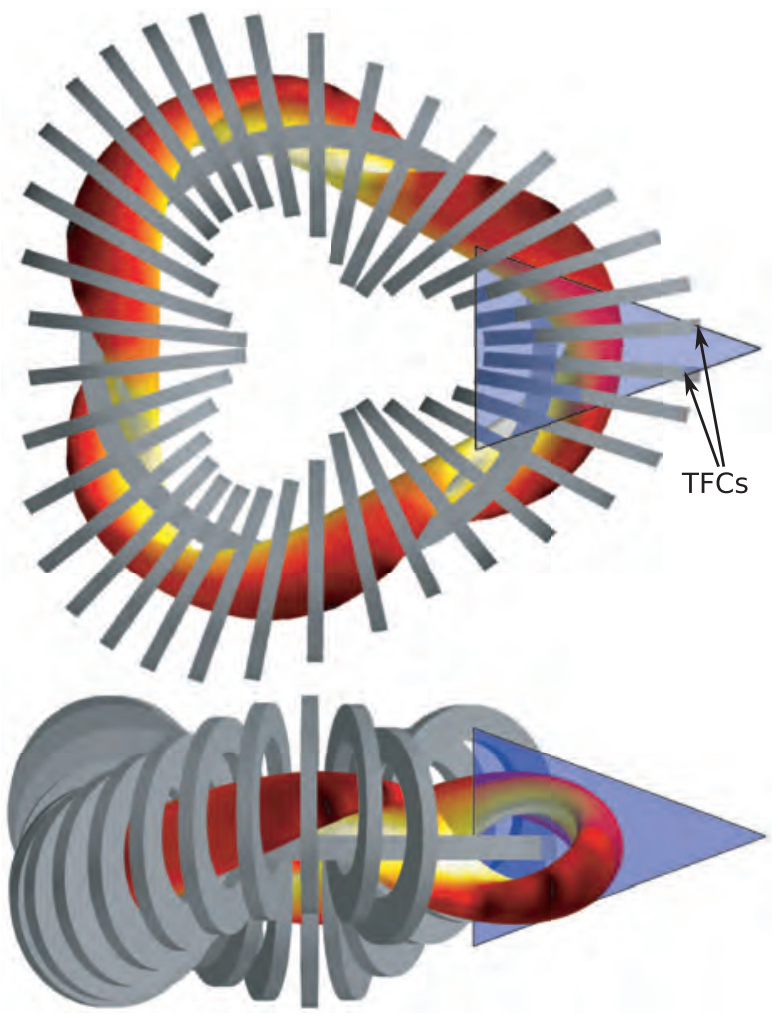

FIG. 5. Details of the camera view. The top plot shows how the toroidal field coils (TFCs) limit the field of view. A typical H-1NF plasma is shown, with the surface colour representing the magnetic field strength. small range of toroidal angles. The images presented here are obtained in impurity carbon ion emission at $514 \mathrm{~nm}$. Because of the relatively cool electron temperatures in $0.5 \mathrm{~T} \mathrm{H} / \mathrm{He}$ plasmas in $\mathrm{H}-1 \mathrm{NF}$, this emission emanates from all regions in the plasma, and so is suitable for revealing the structural details of the mode.

For emission line radiation, the brightness is proportional to the product of the electron and impurity ion densities, and, in our case, only weakly dependent on the electron temperature through the collisional excitation rate coefficient. Mode related fluctuations in temperature and density, which are small compared to the background will therefore result in linear changes to the brightness detected by the camera.

The first and fourth frames in a 16 phase sequence for a $15 \mathrm{kHz}$ mode are shown in Figure 6. The discharges are short but very repeatable, so for this data, each frame was obtained using a separate shot, with the image exposure commencing at the same time in each discharge. Also shown is the DC level across all frames, and the difference between the fourth and first frames. To account for shot-to-shot variability, the images are generally normalized for equal integrated photoelectron counts. A white calibration removes systematic variations in the camera response and a black image is used to
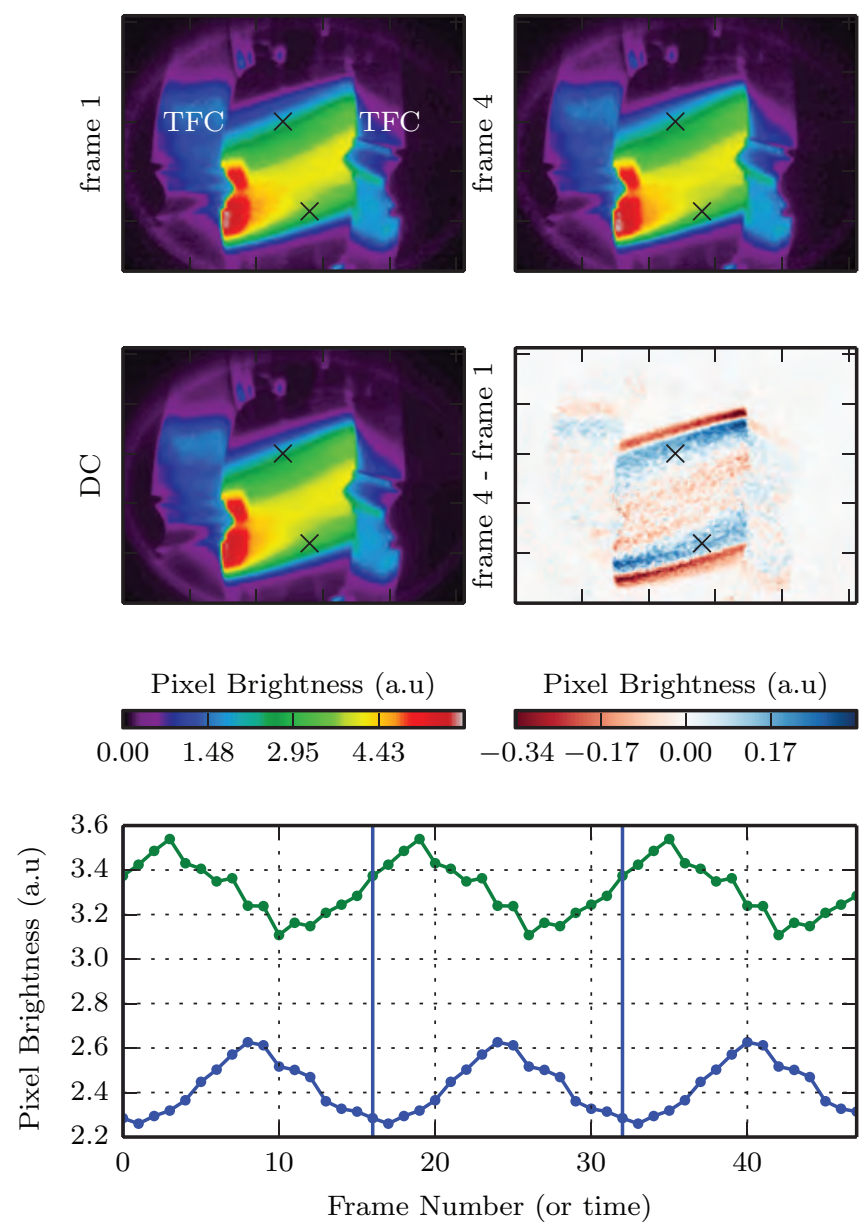

FIG. 6. Top: Frames 1 and 4 from a 16 frame sequence. Middle left: DC component of all 16 frames. Middle right: Difference between frames 1 and 4. Bottom: Evolution of the pixels marked with crosses as a function of frame, or time, showing clear sinusoidal behaviour. The sequence is repeated three times to clearly demonstrate the sinusoidal pattern. 

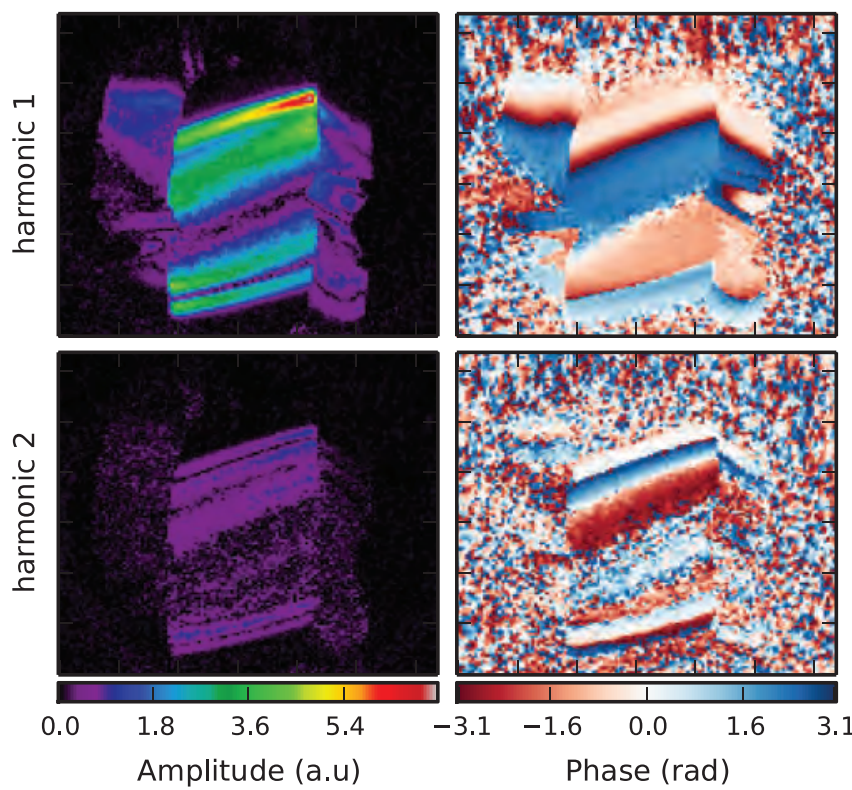

FIG. 7. The amplitude (envelope) and phase of the first two harmonics from the Fourier decomposition of the frames shown in Figure 6.

remove offsets and fixed pattern noise. The intensity variation over a cycle of the pixels marked with crosses is shown in the bottom plot. The 16-frame pattern is repeated three times to make apparent the sinusoidal variation and depth of the first harmonic component intensity modulations.

Figure 7 shows the amplitude (envelope) and phase of the first two harmonics obtained by Fourier processing of the frame sequence shown in Figure 6. Almost all of the power appears in the first harmonic as expected, and the structure of the mode is clear. For this mode, the small amplitude at the center of the projection indicates that this mode has an odd poloidal mode number. This agrees with the toroidal mode number $(n=4)$ and poloidal mode number $(m=-3)$ obtained from analysis of the magnetic probe array data. The slightly different structure of the second harmonic image suggests that this is not an artifact or distortion in the image acquisition process.

Projections of another mode which has been identified magnetically as having mode numbers $n=5$ and $m=-4$ and has a frequency of $20 \mathrm{kHz}$, are shown in Figure 8. This

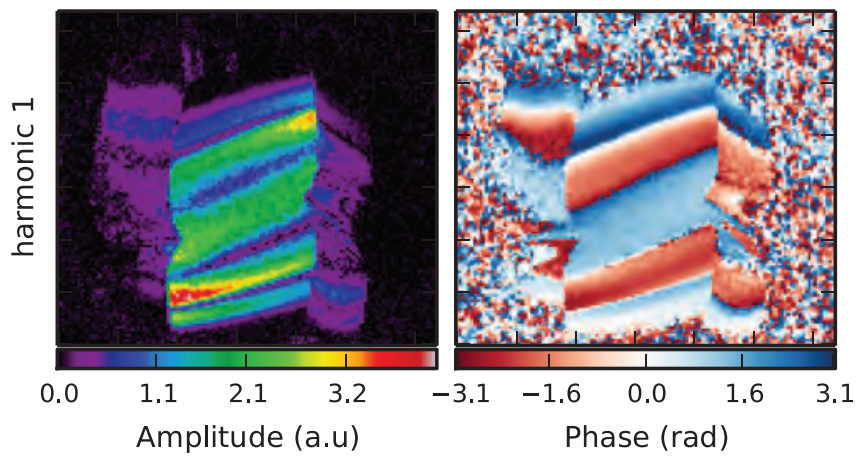

FIG. 8. The amplitude (envelope) and phase of the first harmonic from the Fourier series decomposition of another set of frames from a mode identified as $n=5$ and $m=-4$ using a magnetic probe array. mode has a significantly different structure to the one shown in Figure 7. In this case, the amplitude across the middle is an antinode which is expected for a plasma structure having even poloidal mode number.

\section{CONCLUSION}

By phase locking to a coherent plasma oscillation and synchronously accumulating photons at a fixed phase in the mode cycle, SI is capable of phase-resolved imaging of coherent plasma instabilities with frequencies in the low $\mathrm{kHz}$ to $\mathrm{MHz}$ range. With current camera technology, these images represent millions of lines of sight that can facilitate detailed tomographic reconstruction of the mode radial structure. The utility of the technique has been demonstrated by imaging in $514 \mathrm{~nm}$ carbon ion impurity light, the MHD eigenmodes that appear spontaneously in $0.5 \mathrm{~T} \mathrm{H} / \mathrm{He}$ discharges on the $\mathrm{H}-1 \mathrm{NF}$ heliac.

\section{ACKNOWLEDGMENTS}

The authors would like to thank the H-1NF team for continued support of experimental operations. This work was supported by the Education Investment Fund under the $\mathrm{Su}$ per Science Initiative of the Australian Government. S.R.H. wishes to thank AINSE Ltd. for providing financial assistance to enable this work on H-1NF to be conducted. J.H. and B.B. acknowledge support from the Australian Research Council Discovery, Grant Nos. DP110104833 and DP0666440, respectively.

${ }^{1}$ W. W. Heidbrink, Phys. Plasmas 15, 055501 (2008).

${ }^{2}$ K. L. Wong, R. J. Fonck, S. F. Paul, D. R. Roberts, E. D. Fredrickson, R. Nazikian, H. K. Park, M. Bell, N. L. Bretz, R. Budny, S. Cohen, G. W. Hammett, F. C. Jobes, D. M. Meade, S. S. Medley, D. Mueller, Y. Nagayama, D. K. Owens, and E. J. Synakowski, Phys. Rev. Lett. 66, 1874 (1991).

${ }^{3}$ R. B. White, E. Fredrickson, D. Darrow, M. Zarnstorff, R. Wilson, S. Zweben, K. Hill, Y. Chen, and G. Fu, Phys. Plasmas 2, 2871 (1995).

${ }^{4}$ J. Howard, Rev. Sci. Instrum. 77, 10F111 (2006).

${ }^{5}$ J. Howard, J. Phys. B: At. Mol. Opt. Phys. 43, 144010 (2010).

${ }^{6}$ A. Kirk, B. Koch, R. Scannell, H. Wilson, G. Counsell, J. Dowling, A. Herrmann, R. Martin, and M. Walsh, Phys. Rev. Lett. 96, 185001 (2006).

${ }^{7}$ Z. Linge, M. Takeuchi, N. Nishino, T. Mizuuchi, S. Ohshima, K. Kasajima, M. Sha, K. Mukai, H. Lee, K. Nagasaki et al., Plasma Sci. Technol. 15, 213 (2013).

${ }^{8}$ M. Van Zeeland, J. Yu, M. Chu, K. Burrell, R. La Haye, T. Luce, R. Nazikian, W. Solomon, and W. West, Nucl. Fusion 48, 092002 (2008).

${ }^{9}$ M. Van Zeeland, J. Yu, N. Brooks, W. Heidbrink, K. Burrell, R. Groebner, A. Hyatt, T. Luce, N. Pablant, W. Solomon et al., Plasma Phys. Controlled Fusion 52, 045006 (2010).

${ }^{10}$ J. Yu and M. Van Zeeland, Rev. Sci. Instrum. 79, 10F516 (2008).

${ }^{11}$ J. Yu, M. Van Zeeland, M. Chu, V. Izzo, and R. La Haye, Phys. Plasmas 16, 056114 (2009).

${ }^{12}$ S. Ohdachi, K. Toi, G. Fuchs, S. von Goeler, and S. Yamamoto, Rev. Sci. Instrum. 74, 2136 (2003).

${ }^{13}$ S. Hamberger, B. Blackwell, L. Sharp, and D. Shenton, Fusion Technol. 17, 123 (1990).

${ }^{14}$ B. Blackwell, D. Pretty, J. Howard, R. Nazikian, S. Kumar, D. Oliver, D. Byrne, J. Harris, C. Nuhrenberg, M. McGann et al., preprint arXiv:0902.4728 (2009)

${ }^{15}$ J. Harris, M. Shats, B. Blackwell, W. Solomon, D. Pretty, S. Collis, J. Howard, H. Xia, C. Michael, and H. Punzmann, Nucl. Fusion 44, 279 (2004).

${ }^{16} \mathrm{D}$. Pretty, "A study of MHD activity in the $\mathrm{H}-1$ heliac using data mining techniques," Ph.D. thesis, The Australian National University, 2007. 
${ }^{17}$ D. Pretty and B. Blackwell, Comput. Phys. Commun. 180, 1768 (2009).

${ }^{18}$ S. Ma, J. Howard, B. D. Blackwell, and N. Thapar, Rev. Sci. Instrum. 83, 033102 (2012)

${ }^{19}$ S. Ma, J. Howard, and N. Thapar, Phys. Plasmas 18, 083301 (2011).

${ }^{20}$ J. Bertram, B. Blackwell, and M. Hole, Plasma Phys. Controlled Fusion 54, 055009 (2012).
${ }^{21}$ J. Bertram, M. Hole, D. Pretty, B. Blackwell, and R. Dewar, Plasma Phys. Controlled Fusion 53, 085023 (2011).

${ }^{22}$ S. R. Haskey, B. D. Blackwell, B. Seiwald, M. J. Hole, D. G. Pretty, J. Howard, and J. Wach, Rev. Sci. Instrum. 84, 093501 (2013).

${ }^{23}$ K. V. Mardia and P. E. Jupp, Directional Statistics (Wiley, 2009), Vol. 494.

${ }^{24}$ N. I. Fisher, Statistical Analysis of Circular Data (Cambridge University Press, 1995). 